\title{
Real-time Feedback for Learning the Violin
}

\author{
Rose Johnson \\ The Open University \\ Milton Keynes, UK \\ r.m.g.johnson@open.ac.uk
}

\author{
Janet van der Linden \\ The Open University \\ Milton Keynes, UK \\ j.vanderlinden@open.ac.uk
}

\author{
Yvonne Rogers \\ The Open University \\ Milton Keynes, UK \\ y.rogers@open.ac.uk
}

\begin{abstract}
My PhD looks at how technology can give feedback to novice musicians to help them learn and to encourage them to practice. In particular I am looking at real-time feedback which means that the learner receives feedback while they are playing. This presents both challenges and opportunities, in terms of how feedback can be presented in a useful and motivating way. I intend to explore these through building prototypes and evaluating them in in-the-wild user studies. The outcome of this research will be design guidelines to help future applications of real-time feedback.
\end{abstract}

Real-time feedback, learning violin, motivation, motor-learning, vibrotactile feedback, visual feedback.

\section{INTRODUCTION}

My $\mathrm{PhD}$ is concerned with how real-time feedback can be used to enhance learning the violin. Learning the violin is a difficult task and is thought to take at least 10 years of dedicated practice and training to master (Ericsson et al. 1993). This requires the student to be consistently motivated to practice their instrument. Even when they do practice, there is danger they may not practice in a way their teacher intends. This can slow progress because students have to unlearn mistakes before they can correct their technique.

My research addresses this in a novel way by giving real-time feedback to guide students as they play. Real-time feedback is not used much in traditional teaching methods, but it may have much to offer instrumental training. Firstly feedback can be interpreted in context of the movement that triggered it, setting up a strong link between cause and effect and allowing any information contained in the feedback to refer to this context without the need for additional explanation; whereas in traditional teaching, the learner must interpret a verbal description or demonstration of the movement. Secondly real-time feedback allows the learner to adapt their technique while they play, preventing them from practicing bad technique.

Real-time feedback also presents a number of challenges; the foremost of these being that it is time-dependent and relies on the learner being able to interpret and apply the information contained in the feedback as they play. This means that the way feedback is communicated to the student must be quick to comprehend. This may also place limits on the complexity of information that can be given to a student at any one time.

\section{RESEARCH QUESTION}

The aim of my $\mathrm{PhD}$ is to explore the opportunities that real-time feedback offers for new ways of learning motor-skills by applying it to the specific application of learning the violin. I intend that one of the main outcomes of this exploration will be guidelines for the design of effective systems that use real-time feedback to aid learning. In order to do this I am investigating the following research question:

How can effective real-time feedback be designed to aid learning the violin?

I begin to address this question by first considering what "effective" means in the context of learning the violin. One important aspect of effectiveness is whether the learner's physical movement when playing becomes closer to the desired technique. Another meaning could be a positive effect from the feedback upon the learner's motivation. There is clear evidence that time spent practicing is a strong predictor for musical ability (Sloboda 2005). In order to practice the student must be motivated, whether this comes from an external source such a parent or more internal reason such as an intrinsic enjoyment in playing music. This therefore means that it is important to design feedback with motivation in mind, as a teaching aid that damages a student's motivation would be detrimental, and this may outweigh than any positive effects the tool may have on technique. There could also be other meanings for effectiveness. For example, in a lesson communication between teacher and student is vital; if a feedback system enables teacher and student to communicate more effectively this would also be a positive outcome. It is clear from this discussion that ideas about what makes an effective learning aid are strongly dependent upon its context of use, therefore I place a lot of emphasis upon evaluating real-time feedback in real-use situations. This approach contrasts with much of the related work described in the next section. 


\section{RELATED WORK}

Laboratory studies show that real-time feedback can be effective for enhancing motor-learning. For example Bloomfield and Badler (2008) used realtime vibrotactile feedback to teach karate moves and found that the accuracy of the movement was significantly improved after the training session. Lieberman and Breazeal (2007) have also shown that additional real-time feedback improved the accuracy of participants trying to mimic arm movements of a person on screen.

A small number of projects have looked at giving feedback to musicians using technology. One of these is the iMaestro project ( $\mathrm{Ng}$ et al. 2007) which visualises player and instrument on screen so that they can be viewed from any angle. Other measurements such as bow speed are also displayed as graphs or as a sound. This system has received a positive response from the musicians they worked with; however it is unclear how usable this type of detailed feedback is in realtime. It is possible that this tool was more useful for reviewing a performance after they had finished playing. Additionally the system is limited by the camera based motion capture technology it uses which has prevented it from being taken out of the lab and evaluated in real-world teaching settings. KBow (McMillen) is a more light weight system which uses sensors built into a special bow. This also gives detailed visual feedback in the form of graphs on-screen. However, it is not designed as a learning aid but rather as a way of controlling different sounds or effects as the musician plays. Großhauser and Hermann (2009) describe a system for violin students that gives vibrations as feedback; however no evaluation of this system has been published. These projects show that it is possible to give real-time feedback to novice musicians but that there needs to be more evaluation of such systems to understand how to give real-time feedback in a useful way.

\section{METHODOLOGY}

In order to explore different ways of giving real-time feedback an approach of iterative prototyping is used in which prototypes are repeatedly built and then evaluated. The evaluation stage is used to investigate both how the prototype can be improved but also the more general effect of a particular type of real-time feedback upon the students learning the violin. This method allows the design to evolve in tandem with understanding about how different types of real-time feedback affect learning in the classroom and at home.

As discussed previously, feedback has potential to affect learning in multiple ways, some of which only become salient in real-world real-use settings. For this reason the prototypes are evaluated both in laboratory studies and in in-the-wild deployments over long period of time. In both these settings both quantitative motion capture data and qualitative observation and interview data are collected. The methods used for each individual study are described in more detail in the following sections.

\section{FIRST PROTOTYPE: MUSICJACKET}

I began my research by carrying out studies that looked at the effect of giving real-time vibrotactile feedback to novice musician. I did this by evaluating a prototype system called the MusicJacket. The MusicJacket gives vibrotactile feedback to students as they play to help them correct their bowing movement and the position of their violin. The system measures the player's movement using the Animazoo motion capture suit ${ }^{1}$. This is wirelessly sent to a laptop and analysed to see how far the student's posture and movement deviates from the 'ideal' playing style. The 'ideal' is set at the start of the lesson by the teacher setting the student's posture and guiding the student into the correct bowing movement. Once the motion capture data has been analysed it is turned into the feedback for the student which is controlled by an Arduino ${ }^{2}$ microcontroller which interfaces with the computer via USB. The feedback takes the form of vibrations on the student's arms and torso (see Fig. 1). A push metaphor was originally used to position the motors; this means that if a player feels a vibration he or she should move their arm away from the vibration. The positioning of the motors evolved over the course of the studies in response to comments from participants and specialists about what was most comfortable and easiest to understand.

\section{Evaluation Studies}

The MusicJacket was evaluated in two studies. The first was a week long laboratory study which looked at whether the vibrotactile feedback is successful in changing the way complete beginners bow and hold the violin. Quantitative data (van der Linden et al. 2011a) was collected using the motion capture suit. This showed that participants moved towards the 'ideal' playing style when the feedback is switched on, but whether this then had a lasting effect on their playing was less clear due to the short length of the study. The observation and video data (Johnson et al. 2010) from this study also showed that participants could learn to use the feedback relatively quickly but that it did need explanation from the investigator and time for the participant to experiment with the feedback to understand it properly - it was not intuitive in the sense that participants immediately knew what to

\footnotetext{
${ }^{1}$ http://www.animazoo.com/

${ }^{2}$ http://www.arduino.cc/
} 
do with it and what it was telling them. It also became clear that participants become overloaded if they are given feedback about several things at once. For this reason we restricted ourselves to either giving feedback about violin position or about bowing but not both at the same time.

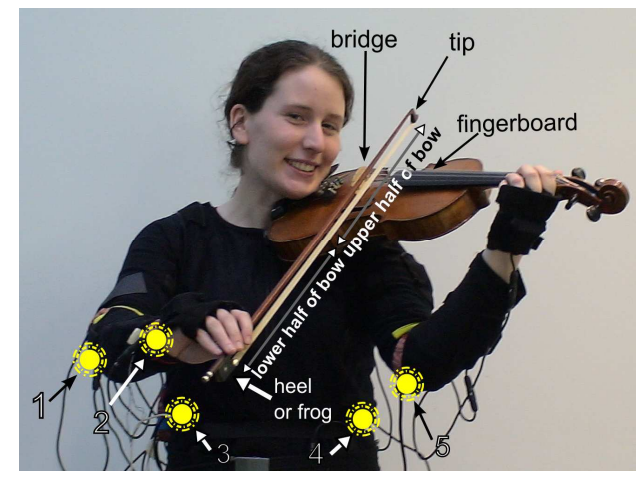

Figure 1: Motor placement in final version of MusicJacket. Motor $1=$ move right arm forward, motor 2 = move right arm back, motors 3, 4, 5 = straighten up and lift violin.

The second study (van der Linden et al. 2011b) took place in more real-world settings. The aim of this study was to find out how real-time feedback might realistically be used in the classroom and whether teachers can incorporate it into their lessons as something useful and relevant to real children learning the violin. We had two groups of participants, one group were aged between 10 and 15 and were taught by their usual teacher in the home of one of the researchers, and the other group were aged between 6 and 9 and were taught by their normal teacher in their normal teaching room at school. All the lessons in the study were one-to-one and the study lasted for two months.

This setting revealed new things about giving realtime feedback. For example we found that the success of the feedback was strongly dependant upon it's relevance to what the child is learning at the time. Children who were given feedback about an issue they were currently working on responded much better to it than those for whom the feedback was an additional area to be working on. For example one of the older children had an issue with using enough bow, which our original system did not cover. The feedback on straight bowing was not effective for her because her focus was on using more bow meaning she did not respond to the feedback when it came on. When the prototype was adapted to encourage her to use more bow, she could respond to the feedback much better.

In these real teaching setting the students were also under much larger cognitive load than in the laboratory study; they had to not only attend to the feedback but also read music and remember their teacher's guidance. In some situations where the cognitive load was too high, for example playing an unfamiliar piece or working on another aspect of technique the participants reported that they had not felt the feedback come on although it had. From this we conclude that feedback must be used in conjunction with task of appropriate difficulty.

\section{SECOND PROTOTYPE}

For the next stage of prototyping the objectives are to make the prototypes more lightweight, affordable and simple enough to use without assistance so that they can be used in home practice. This is in response to interviews we had with teachers after the MusicJacket studies. Another objective at this stage is that it should be flexible enough that the learner and teacher can choose what feedback is given about, so that students are able to adapt the system to their learning goals. This is in response to the findings of the MusicJacket evaluation.

This second set of prototypes is also being used to investigate different ways of giving feedback. Two in particular that will be contrasted at this stage are vibrotactile and visual feedback. Vibrators have an advantage that they can be positioned on the body to communicate movement in a body-centred reference frame and they do not require visual attention which might be needed to read music. However, vibrotactile feedback can only be felt by the student, whereas visual feedback can be seen by both student and teacher or other audience (e.g. parents) this may play a role in terms of intersubjectivity. Investigating feedback in a practice situation also raises issues of whether feedback will affect a student's motivation. It may be that visual feedback will have an advantage in this respect as it has the potential to be attractive as well as informative.

\section{The System}

To make the second prototype light and affordable it is made up of cheaply available sports sweatbands with single sensors sewn onto them (see figure 2). The student wears one of these sensor bands to sense a particular aspect of technique. This arm band then wirelessly sends the readings via an XBee radio link to the feedback component; which is either one of two possible light displays mounted on the musicstand (see figure 3 ) or a vibrotactile arm band. Using these cheap off-the-shelf materials makes each sensor band or feedback display cost approx. £45. The primary cost in both cases is the XBee radio. As well as being cheaper the system is also much simpler to set up than the MusicJacket; it is just a case of switching on the sensor band and the feedback display and it should work straight away.

Any sensor band can be paired with any type of feedback making the system flexible and easily adapted as the student's learning focus changes. Currently sensor bands are being built to sense the 
following: posture of the left arm, correct use of the left wrist, length of bow used, correct hand shape holding the bow. These are all issues mentioned by the teachers that we worked with in the MusicJacket studies. These problems are sensed using accelerometers, bend sensors or gyroscopes as appropriate. Should participants require a different problem to be sensed additional arm bands could easily be made and incorporated into the system.

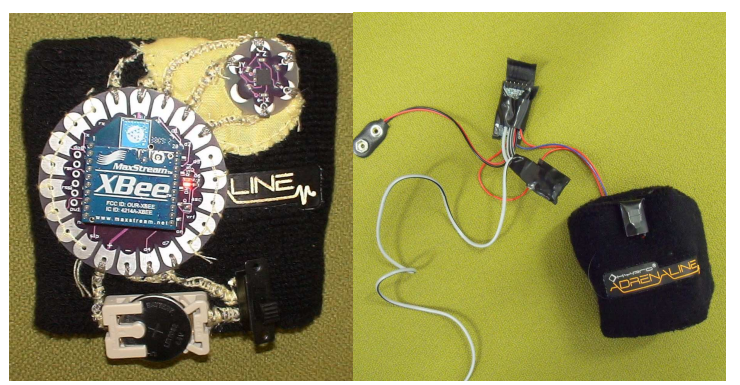

Figure 2: Arm band sensors. Left: uses an accelerometer to sense the posture of left arm. Right: uses a gyroscope to measure the length of bow used.

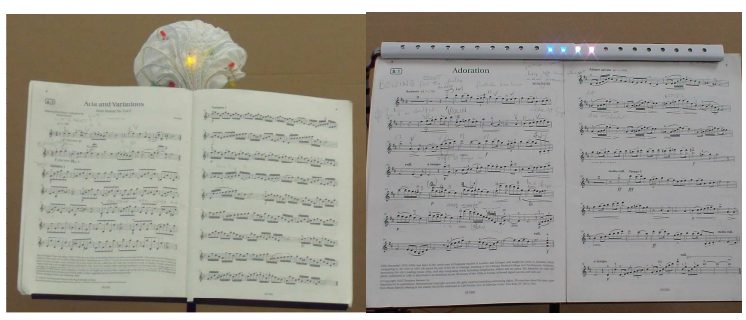

Figure 3: Two ways of giving visual feedback.

\section{Evaluation Studies - Future Work}

These prototypes will be evaluated in two ways. The first study will be short study based in the lab and will be used to test that people can understand and correct their movements using the feedback. This will be evaluated through observation and by capturing motion data using the Animazoo suit from the previous prototype. Interviews and questionnaires will used to find out what styles of feedback are preferred and why.

Based on this study final adjustments will be made to the system before the start of a much larger scale study following the progress of eight novice violinists over several months of practicing with feedback. A month of baseline data will be taken before the study. Data will be collected at regular intervals using the motion capture suit to see whether their playing technique quantitatively improves after the introduction of feedback. Some lessons will also be observed and interviews with student and teacher will be carried out to look for changes in the student's attitude towards playing as well as whether they are finding the feedback useful. To get an idea of any effects upon time spent practicing students will be asked to keep a practice diary for the period of the study. I must, however, be mindful that practice diaries have themselves been shown to increase practice levels and it is like likely that the novelty of the system and simply taking part in the study may also have a similar confounding effect. I hope that by collecting detailed interview data and by collecting many forms of data over long periods of time I will be able to combine these to analyse the subjective and selfreported elements of the data in a way that gives a rounded view of how feedback affects learning.

\section{SUMMARY}

My research looks at innovative ways of facilitating learning by giving real-time feedback to students in response to their movement. In my PhD I look at this in the context of learning the violin, however real-time feedback could have applications in many other areas, for example sports training, posture or physiotherapy. From this research I intend to produce a set of design guidelines which can be used to design systems which give effective and motivating real-time feedback.

\section{REFERENCES}

Bloomfield, A. and Badler, N. (2008) Virtual training via vibrotactile arrays. Presence, 17, 103-120.

Ericsson, K. A., Krampe, R. T. \& Tesch-Römer, C. (1993) The role of deliberate practice in the acquisition of expert performance. Psychological Review, 100(3), 363-363.

Großhauser, T. \& Hermann, T. (2009) Augmented Haptics-An Interactive Feedback System for Musicians Haptic and Audio Interaction Design, Dresden, Germany, 10-11 Sept. 2009, 100-108.

Johnson, R., van der Linden, J. \& Rogers, Y., (2010) MusicJacket: The efficacy of real-time vibrotactile feedback for learning to play the violin. In Extended Abstracts of CHI2010 (WIP) 3475-3480.

Lieberman, J. and Breazeal, C. (2007) TIKL: Development of a wearable vibrotactile feedback suit for improved human motor learning, IEEE Transactions on Robotics, 919-926.

McMillen, K. (2010) Keith McMillen Instruments www.keithmcmillen.com/products/k-bow/ (29\11\10) $\mathrm{Ng}$, K.C., Weyde, T., Larkin, O., Neubarth, K., Koerselman, T. \& Ong, B. (2007) 3d augmented mirror: a multimodal interface for string instrument learning and teaching with gesture support. ICMI2007, Nagoya, Japan, 12-15 Nov., 339-345.

Sloboda, J. (2005) Chapter 19 Music: Where Cognition and Emotion Meet. In Exploring the Musical Mind. OUP. van der Linden, J. Schoonderwalt, E., Bird, J. \& Johnson, R. (2011a) MusicJacket: Combining Motion Capture and Vibrotactile Feedback to Teach Violin Bowing. IEEE Transactions on Instrumentation and Measurement, 60(1) van der Linden, J., Johnson, R., Bird, J., Rogers, Y. \& Schoonderwaldt, E. (2011b) Buzzing to Play: Lessons learned from an in the wild study of realtime vibrotactile feedback. CHI2011, Vancouver, Canada, 7-12 May, to appear. 